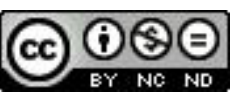

\title{
A Modelagem como um Ambiente de Aprendizagem para a Conversão do Conhecimento Matemático
}

\author{
Modeling as a Learning Environment for the \\ Conversion of Mathematical Knowledge
}

\author{
Milton Rosa* \\ Daniel Clark Orey**
}

\section{Resumo}

A investigação da modelagem como um ambiente de aprendizagem para a construção do conhecimento matemático está se tornando predominante nos meios acadêmicos. Esse ambiente facilita uma estrutura pedagógica que promove a identificação e a disseminação de ambos os conhecimentos, explícito e tácito, dos alunos, através do estudo e da codificação das ideias matemáticas presentes nos sistemas retirados da realidade. Neste artigo, discutimos as principais características da conversão dos conhecimentos matemáticos, explícito e tácito, através da utilização do ambiente de aprendizagem da modelagem.

Palavras-chave: Modelagem Matemática. Conhecimento Tácito. Conhecimento Explícito. Ambiente de Aprendizagem. Conhecimento Matemático.

\footnotetext{
* Doutor em Educação, California State University, Sacramento. Professor do Centro de Educação Aberta e a Distância (CEAD), Universidade Federal de Ouro Preto (UFOP), Ouro Preto, MG, Brasil. Endereço para correspondência: Rua Profa. Zizinha Cruz, 9A, Rosário, CEP: 35400-000. Ouro Preto, Minas Gerais, Brasil. E-mail: milton@cead.ufop.br.

*** Doutor em Educação, University of New México, Albuquerque. Professor do Centro de Educação Aberta e a Distância (CEAD), Universidade Federal de Ouro Preto (UFOP), Ouro Preto, MG, Brasil. Endereço para correspondência: Rua Profa. Zizinha Cruz, 9A, Rosário, CEP: 35400-000. Ouro Preto, Minas Gerais, Brasil. E-mail: oreydc@cead.ufop.br.
} 


\begin{abstract}
Modeling investigation as a learning environment for the construction of mathematical knowledge is becoming prevalent in academic environments. This learning environment facilitates a pedagogical structure that promotes the identification and dissemination of both explicit and tacit student knowledge through the study and codification of mathematical ideas, present in systems taken from reality. In this article, we discuss the main features for the conversion of both explicit and tacit mathematical knowledge through the use of modeling as a learning environment.
\end{abstract}

Keywords: Mathematical Modeling. Tacit Knowledge. Explicit Knowledge. Learning Environment. Mathematical Knowledge.

\title{
1 Introdução
}

De uma maneira geral, o ensino atual da matemática tem provocado preocupações aos professores, aos alunos, aos pais e à sociedade. O campo de pesquisa que trata das questões relacionadas com o ensino-aprendizagem, em especial da matemática, nos diferentes níveis de ensino, tem evoluído muito nas ultimas décadas. As pesquisas e as investigações realizadas na área de Educação Matemática apontam que a matemática ensinada nas escolas, bem como a forma como ela vem sendo ensinada, não acompanharam a evolução social e tecnológica que correspondem às demandas da sociedade atual. Assim, na tentativa de minimizar tais problemas, propostas significativas são apresentadas para a melhoria do ensino que, de acordo com Almeida e Dias (2004, p.20) estão "centradas em enfoques, métodos e estratégias, uma vez que, do ponto de vista teórico, os conteúdos a serem abordados durante as aulas de matemática deverão continuar essencialmente os mesmos".

Desta forma, esperamos que as novas tendências de ensino em Educação Matemática proponham a criação de ambientes de aprendizagem para a matemática, nos quais a atuação do professor seja a de orientador e mediador das atividades propostas nesses ambientes, enquanto que os alunos tenham liberdade de propor, desenvolver, criar, elaborar e modelar as ideias que são necessárias para a construção do próprio conhecimento matemático. Neste sentido, a elaboração de uma proposta educacional significativa, que atenda as novas demandas do ensino, que auxilie a engajar os alunos no processo de ensinoaprendizagem em matemática, que os motive a aprender a aprender e que os ajude a se transformar em cidadãos atuantes na sociedade, é um desafio que se 
impõe a todo sistema educacional.

Portanto, diante deste contexto, entre as várias tendências atuais para o ensino da matemática, acreditamos que aquela que seja a mais adequada e que atenda as necessidades impostas pela sociedade contemporânea é a modelagem matemática. De acordo com Bassanezi (2002), a modelagem pode ser considerada como um dos caminhos pedagógicos que desperta maior interesse, que amplia o conhecimento dos alunos e que os auxilia a estruturar a maneira pela qual eles pensam, raciocinam e agem. Esta tendência de ensino tem como objetivo desenvolver a formação de alunos críticos, reflexivos e que estejam atentos aos diferentes problemas que são enfrentados no cotidiano. No entanto, para que este objetivo seja atingido, existe a necessidade de que os alunos estejam inseridos em um ambiente de aprendizagem que facilite a utilização do conhecimento matemático que eles previamente adquiriram na escola e na comunidade na qual eles estão inseridos.

Desta forma, Barbosa (2001) utilizou a noção de ambiente de aprendizagem apresentada por Skovsmose (2000), e definiu a modelagem como um ambiente de aprendizagem no qual os alunos são convidados a indagar e a investigar, por meio da matemática, situações oriundas de outras áreas da realidade. Contudo, para que os alunos consigam indagar e investigar estas situações, existe a necessidade de que eles utilizem os conhecimentos tácito e explícito, bem como a conversão entre estes conhecimentos, para que eles possam entender, compreender, interpretar e analisar estas situações visando tomar uma posição crítica em relação ao objeto a ser estudado (BASSANEZI, 2002).

Nesse contexto, concordamos com o ponto de vista de Chaves (2000) que apresenta a modelagem como um ambiente de aprendizagem no qual o professor e os alunos assumem determinadas responsabilidades e obrigações pelo desenvolvimento do conhecimento matemático e pela conversão entre os conhecimentos matemáticos tácito e explícito, a partir de situações oriundas, preferencialmente, de suas realidades. De acordo com Moretto (2003), este ambiente de aprendizagem tem como objetivo preparar os alunos para se comportarem como geradores da informação e não como meros acumuladores de dados. Nessa perspectiva, Nonaka e Takeuchi (1997) afirmaram que a informação adquirida, aprendida e apreendida neste ambiente de aprendizagem é transformada em conhecimento que é utilizado pelos alunos, para que eles sejam capazes de modificar as estruturas institucionais, tornando-os aptos na concretização de ações mais efetivas de transformação social.

Assim, em nosso ponto de vista, a modelagem pode ser entendida como 
um ambiente de aprendizagem, que tem como objetivo facilitar a investigação de uma situação-problema através da elaboração de atividades pedagógicas contextualizadas, que auxiliem os alunos na conversão e na utilização dos conhecimentos matemáticos tácito e explícito para a resolução de situaçõesproblema que são propostas nesse ambiente.

\section{0 conhecimento}

Embora o conhecimento seja considerado com um processo necessariamente relacionado com a atividade prática do homem, pois conhecer significa possuir uma representação mental do mundo, o conhecimento também pode ser considerado como a nossa atuação no mundo, a partir da representação que dele temos. Neste contexto, para Turban (1992), o conhecimento integra, analisa e sumariza detalhadamente os dados para produzir, reproduzir e organizar informações úteis, relevantes e atualizadas, que são aplicadas na tomada de decisões, com o objetivo de auxiliar o processo de resolução de problemas. Compartilhando este ponto de vista, Lopez e Donlon (2001) e Roska (2003) afirmam que o conhecimento é uma coleção organizada de dados, fatos, axiomas, leis, regras de inferência e conceitos heurísticos, que são aplicados para resolver situações-problema encontradas no cotidiano e que estão relacionadas com um campo especifico da experiência humana. De acordo com D’ Ambrosio (1993), o conhecimento é originado através de um processo cumulativo de criação, de geração, de organização intelectual, de organização social e de difusão de ideias, conceitos e práticas.

Nesta perspectiva, Rosa (2005) argumenta que o conhecimento está fundamentado na concepção holística, porque busca entender e compreender o relacionamento e o inter-relacionamento entre os componentes dos diferentes sistemas que compõem a realidade. Então, Rosa (2000, p.85) afirma que existe a necessidade de se analisar criticamente o papel de cada um destes componentes, pois esta análise favorece "uma ação transformadora que procura reduzir o grau de complexidade da realidade, através da escolha de um sistema que possa representá-la adequadamente”. De acordo com Bassanezi (2002) e D’Ambrosio (1993), um sistema é uma representação parcial da realidade, que permite aos alunos desenvolverem métodos e estratégias que os auxiliem na explicação, no entendimento, na compreensão e na reflexão sobre os sistemas trabalhados, para que adquiram os conhecimentos necessários que os possibilitem conhecer, lidar, manejar e conviver com a realidade. 


\subsection{Os conhecimentos tácito e explícito}

Frequentemente, procuramos a melhor alternativa para a realização de uma tarefa, pois sabemos qual é o processo de resolução mais adequado para executá-la com eficácia. Neste caso, possuímos as informações necessárias para que possamos decidir, agir e, assim, solucionar os problemas relacionados à tarefa. Algumas vezes, sabemos como resolver um problema, pois possuímos o conhecimento necessário para que sejamos bem-sucedidos em sua resolução. Outras vezes, sabemos quem pode nos ajudar a tomar uma decisão, pois podemos identificar quem é o expert que precisamos para nos auxiliar na resolução de uma determinada situação.

Então, saber qual é a melhor alternativa, saber como resolver um problema e saber quem pode nos auxiliar na tomada de decisão é um processo documentado, formalizado e comunicado através de algum formato explícito, por exemplo, um contrato firmado entre duas pessoas. Neste caso, o conhecimento é caracterizado como explícito. Outras vezes, estas inferências fazem parte do nosso entendimento interno, que está tacitamente enraizado em nosso aprendizado e em nossa experiência, por exemplo, ativar a memória e recorrer às experiências passadas para solucionar um dilema. Nesta situação, o conhecimento é caracterizado como implícito, isto é, o conhecimento é tácito.

Assim, para que um novo conhecimento seja construído, temos que negociar, diariamente, com os nossos conhecimentos explícito e tácito. Por exemplo, quando um novo conhecimento é adquirido, ele se torna parte de nosso conhecimento tácito, o qual é adaptado e, subsequentemente, aplicado na resolução de novos problemas, de novas tomadas de decisão e na realização de novas tarefas. Então, Polanyi (1969) explica que, com a experiência e o aprendizado contínuo, o conhecimento tácito, anteriormente construído, amadurece e evolui em um novo conhecimento, que permanecerá tácito até que seja explicitamente comunicado e transmitido. Neste contexto, de acordo com Nelson e Winter (1982), Kogut e Zander (1992), Nonaka e Takeuchi (1995) e Nonaka, Toyama e Konno (2000), para que o processo de construção do conhecimento seja deflagrado, é importante reconhecer a existência das dimensões explícita e tácita deste conhecimento.

\subsection{O conhecimento tácito}

Esse tipo de conhecimento está embebido na experiência pessoal, é 
subjetivo, contextualizado e análogo (NONAKA; TAKEUCHI, 1997). Por exemplo, um indivíduo não aprende a andar de bicicleta lendo um manual, pois ele necessita da experimentação pessoal e da prática para adquirir as habilidades necessárias para o aprendizado dessa ação. Portanto, esse conhecimento é adquirido e acumulado através da vivência individual, pois envolve fatores intangíveis como crenças, perspectivas, percepções, sistemas de valores, ideias, emoções, normas, pressentimentos e intuições. Desta forma, os alunos constroem o conhecimento tácito ao elaborarem analogias entre os diferentes campos de conhecimento, através de atividades de experimentação, investigação e descoberta e também pelo raciocínio proveniente da análise estruturada do conhecimento obtido em livros (MICHAELSON; MICHIE; BOULANGER, 1985).

No entanto, no ponto de vista de Trentin (2001), a contextualização, a introspecção, a subjetividade e o poder intuitivo do conhecimento tácito são elementos que podem dificultar a articulação do mesmo com a linguagem acadêmica. Contudo, a utilização das sentenças gramaticais e das expressões matemáticas pode favorecer a comunicação e a interpretação sistemática do conhecimento tácito, para que o mesmo possa ser entendido, analisado e transferido para outros contextos. Por exemplo, durante a leitura de um livro, o significado das palavras e o entendimento das regras gramaticais funcionam como um conhecimento tácito, que subsidia o leitor na compreensão do texto. Por outro lado, ao resolver uma situação-problema, os significados dos vocábulos e o entendimento dos símbolos matemáticos auxiliam os alunos na compreensão do problema.

\section{$2.3 \mathrm{O}$ conhecimento matemático tácito}

O conhecimento matemático tácito está relacionado com as maneiras pelas quais os alunos utilizam os conceitos matemáticos e se apropriam das experiências matemáticas, relacionando-as com as próprias experiências, crenças e valores culturais (ERNEST, 1998a). Por exemplo, as mulheres rendeiras do nordeste brasileiro são capazes de confeccionar rendados que possuem conceitos do conhecimento geométrico, por exemplo, a simetria, mas elas não conseguem associar estes princípios com os aspectos matemáticos dessa prática. Todavia, esse conhecimento tácito pode ser expresso formalmente, utilizando-se um sistema de símbolos e regras, através de expressões matemáticas, que podem auxiliar a tradução do conhecimento tácito que as rendeiras possuem para a 
linguagem simbólica da matemática.

De acordo com essa perspectiva, Ernest (1998b) pontua que os principais componentes do conhecimento tácito são o simbolismo e a linguagem matemática, os métodos, as operações simbólicas, as estratégias, os procedimentos e as técnicas que, frequentemente, são aplicáveis na resolução de problemas. Neste contexto, Trentin (2001) aponta que o conhecimento matemático tácito pode ser explicitamente comunicado, representado e demonstrado com a utilização de uma linguagem matemática simbólica, estruturada e sistematizada, isto é, através da elaboração dos modelos matemáticos.

\section{$2.4 \mathrm{O}$ conhecimento explícito}

O conhecimento explícito é sistematicamente transmitido, comunicado e compartilhado, pois se expressa através das palavras, dos números, dos dados, das fórmulas matemáticas e dos procedimentos codificados (NONAKA; TAKEUCHI, 1997). De modo geral, este tipo de conhecimento é formalizado através de conceitos, textos, desenhos e diagramas; pode, também, ser articulado na linguagem formal, incluindo as sentenças gramaticais e as expressões matemáticas. Por exemplo, na leitura de um livro, a atenção do leitor está focalizada nos caracteres das palavras, isto é, no significado explícito do texto. Por outro lado, na demonstração de fórmulas matemáticas, o conhecimento é explicitamente expresso através da utilização de princípios e conceitos matemáticos.

Desta forma, o conhecimento explícito está relacionado com um fato concreto, que pode ser disseminado pelo professor através da utilização dos livros didáticos, do conhecimento acadêmico sobre a disciplina, do conhecimento das práticas instrucionais pedagógicas e por qualquer outro método de utilização de materiais e instrumentos tecnológicos, pois estes elementos auxiliam a absorção, a internalização e, consequentemente, a transferência e a aplicabilidade deste conhecimento para outras áreas do conhecimento humano.

\subsection{0 conhecimento matemático explícito}

O conhecimento matemático explícito refere-se ao conhecimento transmissível em linguagem formal e sistemática, pois este conhecimento é objetivo, racional, sequencial e teórico; sendo frequentemente codificado com a utilização de regras, fórmulas e equações matemáticas, através da utilização da linguagem 
e do simbolismo matemático. Nonaka e Takeuchi (1997) explanam que o conhecimento matemático explícito é comunicado pela linguagem acadêmica proposicional, e é demonstrado por outras representações simbólicas como as definições, as hipóteses, as conjeturas, os axiomas, os teoremas e as teorias. Para Frade (2004), os raciocínios lógico e dedutivo, as provas formais e informais, a resolução de problemas e a análise computacional também são componentes do conhecimento matemático tácito. De acordo com Trentin (2001), o conhecimento matemático explícito pode ser formalizado através dos teoremas e das teorias e pela utilização de equações e fórmulas matemáticas e físicas, pois estes elementos facilitam o manuseio dos modelos matemáticos, que são elaborados no ambiente de aprendizagem da modelagem. Por exemplo, a figura 1 mostra como o conhecimento matemático explícito pode ser utilizado para provar o Teorema de Pitágoras.

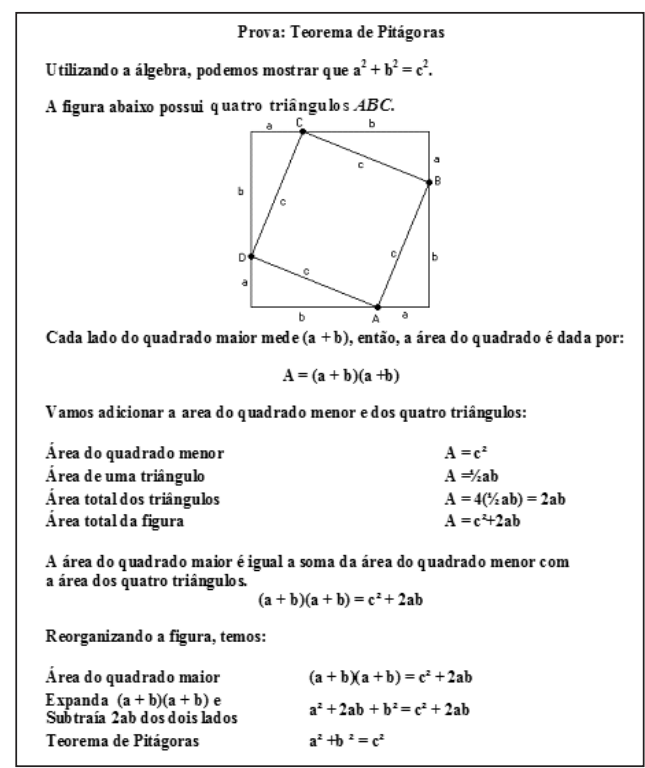

Figura 1- Utilizando o conhecimento matemático explícito para provar o Teorema de Pitágoras

Os manuais, os pôsteres e os relatórios também podem ser considerados como maneiras de sistematizar o conhecimento matemático explícito, pois os alunos têm que comunicar os resultados obtidos na resolução de situaçõesproblema através da análise dos modelos, descrevendo-os, representando-os, interpretando-os e validando-os matematicamente. 


\section{Os modelos matemáticos}

De modo geral, um modelo é a representação de uma ideia, de um conceito, de um objeto ou de um fenômeno (GILBERT, BOULTER; ELMER, 2000). De acordo com Greca e Moreira (2002), os modelos matemáticos podem ser considerados como ferramentas pedagógicas elaboradas para facilitar o entendimento e a compreensão do funcionamento de sistemas que são retirados da realidade. Neste sentido, Ornek (2008) refere-se aos modelos matemáticos como representações externas, precisas e consistentes com o conhecimento científico que é socialmente construído e compartilhado por um determinado grupo social.

Diante desta perspectiva, o objetivo da elaboração dos modelos matemáticos é explicitar o conhecimento tácito através da tradução dos modelos mentais, que foram previamente formulados através das experiências vivenciadas pelos alunos. Por exemplo, Knijnik (1996) propôs atividades sobre a demarcação da terra a partir de um trabalho de pesquisa realizado junto aos participantes do Movimento dos Sem Terra (MST). A atividade realizada enfocou o método da cubação da terra, que é uma prática tradicionalmente utilizada pelos integrantes deste movimento. Flemming; Luz; Collaço de Mello (2005, p.41) definem o termo cubação de terra como a existência de "problemas de medição de áreas de terrenos com formas diversas".

Então, a utilização da prática de cubação da terra como proposta pedagógica para a realização de atividades para o ensino-aprendizagem da matemática mostra a importância da contextualização de situações-problema, no ambiente de aprendizagem da modelagem, para que o conhecimento matemático tácito seja explicitado através da utilização de modelos. Assim, vamos utilizar o exemplo apresentado por Flemming, Luz e Collaço de Mello (2005, p.42) para "calcular a área de terras com formato quadrangular que mede 114 metros x 152 metros x 90 metros x 124 metros".

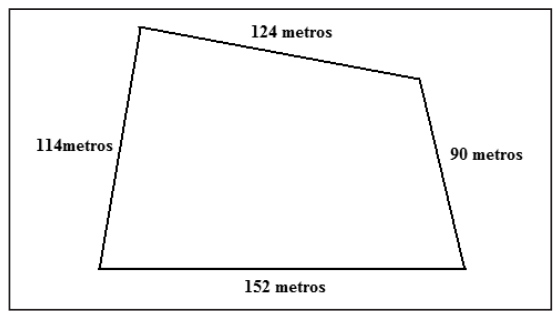

Figura 2 - Figura geométrica com formato quadrangular irregular 
Então, o conhecimento tácito dos integrantes do movimento MST pode ser representado pelo modelo mental que transforma "a forma do terreno dado num [retângulo] de 138 metros x 102 metros. Portanto numa área de 14076 metros quadrados" (FLEMMING; LUZ; COLLAÇO DE MELLO, 2005, p. 42).

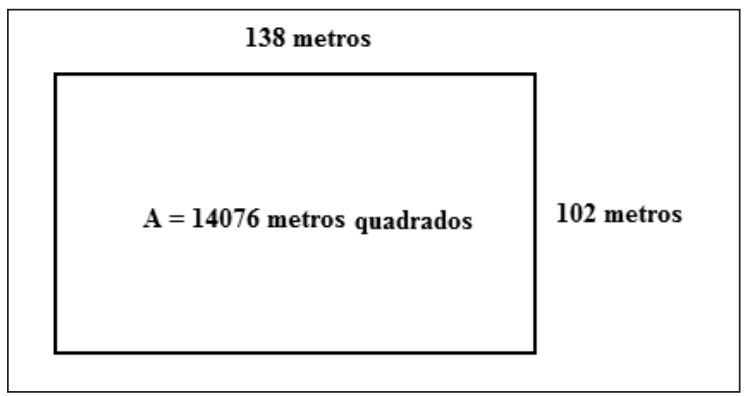

Figura 3 - Figura geométrica transformada em um retângulo

Podemos explicitar o modelo mental deste conhecimento tácito através do seguinte modelo matemático:

- Vamos transformar o quadrilátero irregular em um retângulo cuja área pode ser facilmente determinada através da aplicação da fórmula $A=b \bullet h$.

- Para determinarmos as dimensões do retângulo, devemos calcular a média aritmética dos dois lados opostos do quadrilátero irregular. Assim, temos que:

$$
\begin{aligned}
& \text { Base }=\frac{152+124}{2}=138 \text { metros } \\
& \text { Altura }=\frac{114+90}{2}=102 \text { metros }
\end{aligned}
$$

- Para determinarmos a área desta figura irregular, devemos determinar a área do retângulo. Assim, temos que:

$$
\begin{aligned}
& A=b \bullet h \\
& A=138 \bullet 102 \\
& A=14076 m^{2}
\end{aligned}
$$

Com relação a esta situação-problema, outro modelo mental, proveniente do conhecimento tácito, também pode ser explicitado através de um modelo 
matemático. De acordo com Flemming, Luz e Collaço de Mello (2005, p.42), o terreno irregular apresentado neste exemplo também pode ser transformado em "um quadrado de lado 120 metros, portanto com área de 14400 metros quadrados. Neste sentido, podemos observar "que o valor de 120 foi calculado fazendo a soma das dimensões dividido por quatro".

Para Bassanezi (2002, p.24), um modelo "é eficiente a partir do momento que nos conscientizamos que estamos sempre trabalhando com aproximações da realidade, ou seja, que estamos elaborando representações de um sistema ou parte dele". Assim, Flemming, Luz e Collaço de Mello (2005, p. 42) explicam que, "do ponto de vista formal da Matemática, ambos os métodos apresentam o cálculo aproximado da área que satisfazem plenamente as necessidades e a história de vida dos integrantes do grupo".

De acordo com Biembengut (2004), o modelo elaborado neste exemplo constitui um conjunto de símbolos e relações matemáticas que interagem entre si para traduzir um fenômeno a ser estudado ou um problema proveniente de uma situação real. Assim, o modelo pode ser considerado como uma "expressão das percepções da realidade, do desejo, da aplicação e da representação" (BIEMBENGUT, 2004, p. 17). Neste sentido, Bassanezi (2002, p.24) pontua que "a modelagem consiste, essencialmente, na arte de transformar situações da realidade em problemas matemáticos cujas soluções devem ser interpretadas na linguagem usual". Então, a elaboração de modelos no ambiente de aprendizagem da matemática, "requer uma série de procedimentos que passam pela observação cuidadosa da situação ou do fenômeno a ser modelado, pela interpretação da experiência realizada, pela captação do significado do que produz" (BIEMBENGUT, 2004, p. 17).

\section{Um ambiente de aprendizagem para a conversão do conhecimento matemático}

Para Ausubel, Novak e Hanesian (1980), os alunos constroem o conhecimento matemático com base no conhecimento que foi previamente estruturado. Para eles, um dos fatores mais importantes para o ensinoaprendizagem em matemática é a utilização do conhecimento prévio acumulado pelos alunos. De acordo com Portier e Wagemas (1995), o conhecimento matemático prévio é formado pelos conhecimentos explícito e tácito, pois este conhecimento é dinâmico, estruturado e está disponível, para os alunos, antes que uma atividade de aprendizagem seja realizada. 
Considerando este contexto, a construção do conhecimento matemático e a utilização do conhecimento prévio dos alunos podem ser favorecidas se o ensino-aprendizagem em matemática acontecer em um ambiente de aprendizagem que contemple a natureza tácita e explícita do conhecimento (ERNEST, 1998a). Nesta perspectiva, Nonaka (1991) enfatiza que, nesse ambiente de aprendizagem, o conhecimento é compartilhado através das interações interpessoais e intrapessoais, nas quais os alunos utilizam o conhecimento tácito previamente acumulado, para demonstrá-lo explicitamente através de atividades pedagógicas contextualizadas.

Entretanto, em nosso ponto de vista, um ambiente de aprendizagem somente será propício para o ensino-aprendizagem em matemática se o modelo pedagógico nele utilizado for estruturado para facilitar a análise critica dos dados, favorecer a manipulação adequada da informação e promover a construção e a transferência do conhecimento matemático, através das interações sociais entre os participantes desse processo. Em acordo com as afirmações de Consway e Whittingham (2001) e Trentin (2001), os quais postulam que o conhecimento matemático é construído em um ambiente de aprendizagem no qual os modos diferenciados de resolução de problemas são compartilhados entre os participantes deste processo de ensino.

Em coerência com essa perspectiva, acreditamos que a modelagem matemática pode ser considerada como um ambiente de aprendizagem que facilita a construção e a transferência do conhecimento matemático, através da utilização dos conhecimentos explícito e tácito que interagem neste ambiente.

\subsection{A modelagem matemática}

É impossível a tentativa de localizar no tempo e no espaço a primeira vez em que foram expressos os interesses e a preocupação em relação à modelagem matemática. Entretanto, a modelagem se manifesta desde os tempos mais remotos, através de situações isoladas e pouco sistematizadas, nas quais a humanidade utilizou o conhecimento matemático para entender e compreender os fenômenos da vida cotidiana.

Qualquer situação da vida diária pode ser traduzida em uma linguagem matemática, ou seja, estes aspectos podem, de alguma forma, ser matematizados. Assim, um dos principais objetivos da modelagem é auxiliar os alunos compreenderem as relações e as conexões da matemática com a vida cotidiana (ROSA; OREY, 2008). Como a matemática tem uma linguagem e um modo de 
pensar próprio, temos, também, como objetivo, levar os alunos ao entendimento desta linguagem e à aplicação do raciocínio matemático na resolução de situações-problema contextualizadas.

Neste contexto, Barbosa (2002) e Rosa e Orey (2007) argumentam que a modelagem pode ser considerada como um ambiente de aprendizagem que pode auxiliar os alunos na matematização, na compreensão e na análise de determinados fenômenos que estão presentes no cotidiano. No entanto, para compreender e resolver uma situação-problema, os alunos devem acessar os conhecimentos matemáticos tácito e explícito previamente adquiridos através da elaboração de modelos matemáticos, num ambiente de aprendizagem que favoreça a indagação, a investigação e a reflexão. De acordo com esta perspectiva, Burak (1992, p.62) desvela que a modelagem "constitui-se em um conjunto de procedimentos cujo objetivo é estabelecer um paralelo para tentar explicar, matematicamente, os fenômenos presentes no cotidiano dos alunos", ajudando-os a refletir sobre o resultado obtido, a fazer previsões e a tomar decisões em relação ao objeto de estudo, como também postula Bassanezi (2002).

\subsection{A modelagem matemática como um ambiente de aprendizagem}

A análise crítica dos fenômenos e dos problemas enfrentados pela comunidade escolar serve para ativar a criatividade dos alunos e melhorar o desempenho matemático dos mesmos, através da resolução de situaçõesproblema contextualizadas, motivadoras e desafiadoras (BECKMAN, 1997). No entanto, para que os alunos sejam criativos, eles precisam "utilizar ambientes de aprendizagem que proporcionem a motivação necessária para que eles possam desenvolver e exercer a capacidade crítica que possuem, através da análise crítica da geração e produção do conhecimento" (ROSA; OREY, 2007, p.202). Dessa forma, concebemos a modelagem como um ambiente de aprendizagem que pode facilitar a construção do conhecimento matemático, pois esse ambiente promove a construção, a acumulação, a manipulação e a disseminação do conhecimento matemático (BARBOSA, 2007; ROSA; OREY, 2007).

De acordo com esta perspectiva, Barbosa (2002) explica que a modelagem pode ser considerada como um ambiente de aprendizagem que favorece a investigação de outras áreas do conhecimento através da elaboração dos modelos matemáticos. Assim, nesse ambiente, os alunos são convidados a indagar e a investigar, através da matemática, situações-problema presentes nos sistemas que são retirados da realidade (BARBOSA, 2007). Porém, para 
que este aspecto da aprendizagem ocorra satisfatoriamente, Rosa e Orey (2007, p.7) explanam que os alunos devem utilizar "fórmulas, gráficos e um grupo de expressões matemáticas ou representações diversas para auxiliar a resolver e analisar uma determinada situação-problema". Isso significa que os alunos devem utilizar a linguagem matemática simbólica para descrever o comportamento abstrato dos sistemas a serem estudados (ROSA; OREY, 2003). Assim, concordamos com Chaves (2000) acerca de que o ambiente de aprendizagem proporcionado pela modelagem oportuniza aos alunos a construção do conhecimento matemático. Nesse sentido, a construção do conhecimento matemático deve ser mediada pelo professor, através das interações sociais, para que os alunos possam utilizar e converter os conhecimentos matemáticos tácito e explícito valendo-se de atividades matemáticas contextualizadas.

Entendemos que o ambiente de aprendizagem da modelagem é dinâmico, pois favorece a elaboração de modelos matemáticos através de práticas discursivas que se desencadeiam a partir de espaços de interações sociais entre aluno-aluno, professor-aluno e aluno-professor (BARBOSA, 2007; ROSA; OREY, 2007). Estas práticas discursivas contribuem para que os alunos desenvolvam o raciocínio crítico através de discussões matemáticas reflexivas, que tem como objetivo utilizar os objetos discursivos da linguagem matemática, por exemplo, os símbolos, as notações, os diagramas, os termos, os conceitos, as noções, as definições, os axiomas, as teorias e os teoremas (KITCHER, 1984; ROSA; OREY, 2008) para a construção do conhecimento matemático. Em outras palavras, o professor e os alunos codificam o conhecimento tácito em explícito com a utilização dos objetos matemáticos através da elaboração de modelos.

Neste contexto, o ambiente de aprendizado da modelagem favorece um espaço pedagógico no qual os alunos compartilham e transmitem os conhecimentos explícito e tácito, que são construídos e acumulados, nesse ambiente, através de atividades significativas. Para Ausubel, Novak e Hanesian (1978, p.159) "o aprendizado significativo acontece quando uma informação nova é adquirida mediante um esforço deliberado por parte do aluno em conectar a nova informação com os conceitos ou com as proposições relevantes que preexistem em sua estrutura cognitiva". De acordo com Ford et al. (1991), esses conceitos ou proposições são definidos como registros de fenômenos que foram associados a um específico sistema retirado da realidade. Entendemos que, na aprendizagem significativa, os alunos adquirem as informações e as habilidades necessárias para a resolução de situações-problema através da interação dos mesmos com os demais alunos e com os professores que estão envolvidos no processo de ensino-aprendizagem, deflagrado no ambiente de 
aprendizagem da modelagem. Nesse sentido, Sorensen e Lundh-Snis (2001) e Mclnerney (2002) propõem que os professores devem entender a modelagem como um ambiente de aprendizagem, para que possam desenvolver práticas discursivas e as atividades colaborativas, pois é nesse ambiente de aprendizagem, em conjunção com atividades pedagógicas de investigação, que ocorre a conversão entre os conhecimentos explícito e tácito. Neste processo, de acordo com Rosa e Orey (2007, p. 203), “o papel dialógico e mediador do professor é de suma importância".

Então, o ambiente de aprendizagem da modelagem auxilia a desenvolver nos alunos a capacidade de codificar, armazenar, converter e utilizar os conhecimentos tácito e explícito, pois eles adquirem esses conhecimentos em múltiplas fontes, disponibilizando-os e representando-os em forma de modelos. A interação social que é desencadeada no ambiente de aprendizagem da modelagem também possibilita que os conhecimentos explícito e tácito possam ser processados com criatividade, pois esse ambiente favorece o engajamento dos alunos em atividades dinâmicas, motivadoras e desafiadoras, facilitando a internalização e a externalização desses conhecimentos através da interação social (CHOO, 1996; KERMALLY, 2002) e de atividades pedagógicas diversificadas, tais como seminários, fóruns de discussão, reuniões, mesasredondas, conversas, diálogos e apresentação de relatórios (SORENSEN; LUNDH-SNIS, 2001).

\section{A conversão entre os conhecimentos explícito e tácito no ambiente de aprendizagem da modelagem}

Leonard e Sensiper (1998) descrevem o conhecimento como sendo um continuum. Em um extremo do continuum, o conhecimento é subjetivo e experimentalmente construído; isto é, o conhecimento é tácito. No extremo oposto, o conhecimento é codificado, racional, acessível e estruturalmente construído; isto é, o conhecimento é explícito. Depreendemos, então, que o conhecimento evolui, naturalmente, entre os dois extremos do continuum, através das conversões que ocorrem entre os conhecimentos explícito e tácito. De acordo com Frade (2004), a conversão entre esses conhecimentos está situada na prática social e se move no continuum, do conhecimento tácito para o explícito e do explícito para o tácito.

Polanyi (1969) refere que a conversão entre os conhecimentos explícito e tácito é importante para o processo de construção do conhecimento, pois apesar de eles serem conhecimentos opostos, se complementam e se relacionam. 
Nonaka (1991) aceita tal ponto de vista, pois para ele, esses conhecimentos são mutuamente complementares, isto é, interagem entre si no processo criativo da construção de novos conhecimentos. A nosso ver, a interdependência entre os conhecimentos tácito e explícito permite o trânsito entre eles, bem como a conversão de um conhecimento para outro.

Nonaka (1991) denomina o processo de interação entre os conhecimentos explícito e tácito como conversão do conhecimento. De acordo com Nonaka (1994), Nonaka e Takeuchi (1995), Choo (1996), Malhotra (2000) e Choi e Lee (2002), existem quatro categorias que favorecem a conversão entre os conhecimentos explícito e tácito, ou seja, a socialização, a externalização, a internalização e a combinação. O Quadro 1 mostra as quatro categorias do processo de conversão do conhecimento.

\begin{tabular}{|c|c|c|}
\hline $\begin{array}{c}\text { Do conhecimento } \\
\text { tácito }\end{array}$ & $\begin{array}{c}\text { Para o conhecimento } \\
\text { tácito }\end{array}$ & $\begin{array}{c}\text { Para o conhecimento } \\
\text { explícito }\end{array}$ \\
\hline $\begin{array}{c}\text { Do conhecialização } \\
\text { explícito }\end{array}$ & Internalização & Externalização \\
\hline
\end{tabular}

Quadro 1 - As quatro categorias do processo de conversão do conhecimento

Nesta perspectiva, Nonaka e Takeuchi (1995) expõem que o processo de conversão entre os conhecimentos explícito e tácito é espiralado, pois é um processo contínuo e dinâmico que evolui através das interações sociais. Figura 4 mostra a espiral da construção do conhecimento.

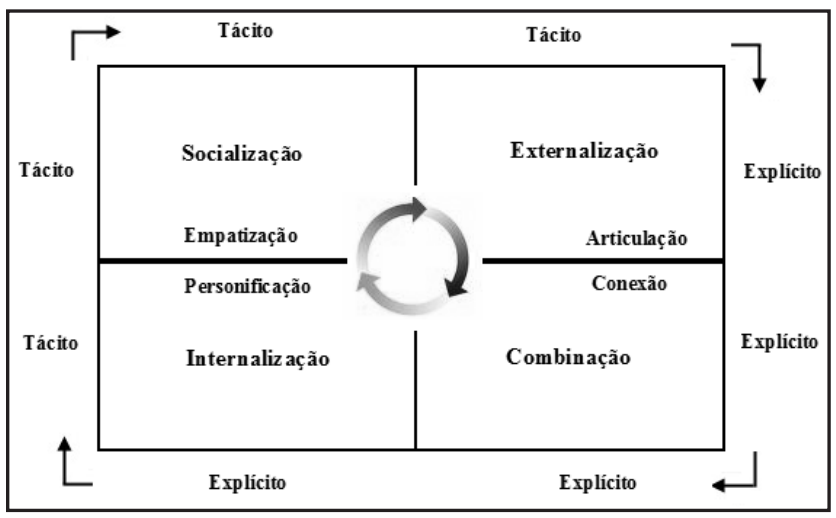

Figura 4- Espiral da construção do conhecimento 


\section{Os processos de conversão entre os conhecimentos explícito e tácito}

A seguir, descrevemos brevemente os quatro processos de conversão que ocorrem entre os conhecimentos tácito e explícito.

\subsection{A socialização do conhecimento: do tácito para o tácito}

Neste processo, o professor e os alunos compartilham o conhecimento tácito através das experiências, das ideias, dos modelos mentais e das habilidades técnicas através da elaboração de atividades interativas, cooperativas e contextualizadas. As atividades de observação, de experimentação, de investigação e de simulação, bem como as interações discursivas são processos pedagógicos que permitem a transferência mútua da informação, favorecendo a socialização do conhecimento entre os professores e os alunos e entre os alunos e os alunos, através do ambiente de aprendizagem da modelagem.

\subsection{A internalização do conhecimento: do explícito para o tácito}

Uma atividade que é baseada no conhecimento explícito pode ser modificada com a utilização de uma atividade fundamentada no conhecimento tácito. Isto significa que o conhecimento interpessoal é transformado em conhecimento intrapessoal. Neste processo, a reflexão interna e a troca de informações entre o professor e os alunos e entre os alunos e os alunos favorecem a internalização do conhecimento, facilitando o desenvolvimento da consciência crítica através dos relacionamentos sociais. Deste modo, a internalização do conhecimento está relacionada com o aprender fazendo, que é um processo pedagógico que caminha do plano social (das relações interpessoais) para o plano individual (das relações intrapessoais).

Nesse processo, os alunos são personagens ativos no ambiente de aprendizagem da modelagem, pois eles constroem os próprios conhecimentos a partir de relações interpessoais e intrapessoais que ocorrem nesse ambiente. Nonaka, Toyama e Konno (2000) apontam que no processo de internalização, o conhecimento explícito dos alunos pode ser convertido em conhecimento tácito através da utilização de atividades pedagógicas de simulação, de experimentação, de investigação, e, também, através da elaboração de modelos matemáticos. 


\subsection{A externalização do conhecimento: do tácito para o explícito}

A característica mais importante deste processo é a articulação entre os conhecimentos tácito e explícito através da utilização das metáforas, das analogias, dos conceitos, das hipóteses e dos modelos. No entanto, é importante salientar que quando os alunos conceituam uma imagem, eles expressam a essência desta imagem através da linguagem formal e acadêmica. Nesse sentido, Nonaka e Takeuchi (1996) explanam que, através do diálogo e da reflexão coletiva, os alunos utilizam as analogias e as metáforas que são mais adequadas para a construção do conhecimento matemático, através da utilização de conceitos matemáticos que estão implícitos no conhecimento tácito e que são explicitados no processo de externalização desse conhecimento.

\subsection{A combinação do conhecimento: do explícito para o explícito}

Os diferentes tipos de conhecimento explícito que os alunos possuem são combinados e convertidos em um novo conhecimento explícito, que contém um nível mais elevado de complexidade. Esse processo permite que os conhecimentos explícitos, que são combinados, sejam reorganizados, reestruturados, sistematizados e refinados. No ambiente de aprendizagem da modelagem, os alunos compartilham diferentes conhecimentos explícitos, baseados em aprendizados prévios, integrando-os num conhecimento explícito mais elaborado, criando, dessa forma, um novo conhecimento explícito. De acordo com Choo (1998), essa é uma forma de aprendizado típica da estrutura pedagógica do ensino da matemática, que deve estar presente no ambiente de aprendizagem da modelagem.

\subsection{O conhecimento matemático e a conversão entre os conhecimentos no ambiente de aprendizagem da modelagem}

A construção do conhecimento matemático está associada com as oportunidades oferecidas pelo ambiente de aprendizagem da modelagem, pois esse ambiente pode contribuir de maneira satisfatória para que os professores e os alunos compartilhem, transfiram e convertam os conhecimentos matemáticos tácito e explícito, através da utilização de atividades pedagógicas significativas, colaborativas e contextualizadas. Nesse sentido, a construção do conhecimento matemático é um processo interativo, contínuo e sinérgico (CHOO, 1996; 
BLOODGOOD; SALISBURY, 2001) que tende a facilitar a conversão entre os conhecimentos explícito e tácito que é desencadeada no ambiente de aprendizagem da modelagem.

Então, o ambiente de aprendizagem da modelagem é propício para o processo de internalização do conhecimento matemático, pois esse ambiente permite que os alunos utilizem as experiências formais (acadêmicas) e a experiências informais (cotidianas) que foram adquiridas durante o processo de ensino e aprendizagem nos ambientes educacionais formal e informal. Desta forma, o ambiente de aprendizagem da modelagem facilita a apropriação das experiências matemáticas através da utilização dos procedimentos e das técnicas que são necessárias para a elaboração dos modelos, para que, posteriormente, o conhecimento matemático tácito seja devidamente externalizado. Nesse contexto, a modelagem pode ser considerada como um ambiente de aprendizagem que facilita a externalização do conhecimento matemático tácito, pois favorece a racionalização do conhecimento tácito, articulando-o com o conhecimento explícito através da elaboração dos modelos matemáticos.

Sendo assim, ressaltamos que o ambiente de aprendizagem da modelagem favorece o fluxo socialização $=>$ externalização $=>$ combinaçã $o=>$ internalização para a conversão entre os conhecimentos explícito e tácito. Por exemplo, o fluxo direto de conversão entre os conhecimentos tácito e explícito envolvendo a socialização e a combinação se torna possível por intermédio do ambiente de aprendizagem da modelagem. A Figura 5 mostra as interações entre as quatro categorias que favorecem a conversão entre os conhecimentos tácito e explícito no ambiente da modelagem matemática.

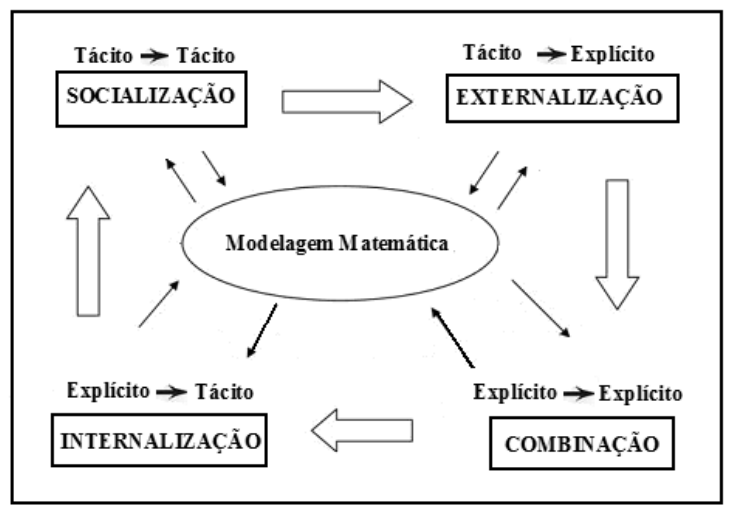

Figura 5 - A conversão entre os conhecimentos tácito e explícito 


\section{A elaboração de modelos no ambiente de aprendizagem da matemática: o Problema Babilônio}

Vamos exemplificar o relacionamento entre os conhecimentos explícito e tácito no ambiente de aprendizagem da modelagem ao traduzir um aspecto retórico do conhecimento matemático babilônio (ROSA, 2007) para a matemática acadêmica, através da elaboração de modelos matemáticos que podem auxiliar os alunos na determinação das dimensões de figuras quadrangulares e retangulares.

Convém observar que, apesar do avanço que os babilônios tiveram nas ciências e na matemática, a aritmética e a álgebra que eles usavam para a resolução de problemas eram retóricas e, muitas vezes, elementares, pois eles utilizavam, persuasivamente, uma linguagem complexamente enfática. Assim, considere o seguinte problema que foi escrito numa das tábuas pertencentes ao período babilônio (JOSEPH, 2000, p.110).

O comprimento de um retângulo excede a sua largura em sete unidades. A área do retângulo é de 60 unidades quadradas. Determine o comprimento e a largura do retângulo.

Devemos observar que, na tábua babilônia, o problema foi escrito em base sexagesimal, que era a base numérica utilizada pelos babilônios. Para efeitos didáticos, este problema foi traduzido para a base decimal. Assim, de acordo com Joseph (2000), a solução fornecida pelos babilônios é a seguinte:

- Determine a metade do valor em que o comprimento do retângulo excede a largura.

- O resultado é 3,5.

- Multiplique 3,5 por 3,5.

- O resultado é 12,25.

- Adicione 60 e 12,25.

- O resultado é 72,25.

- Determine a raiz quadrada de 72,25.

- O resultado é 8,5.

Agora, proceda da seguinte forma:

- Subtraia 3,5 de 8,5

- Adicione 3,5 a 8,5

- O comprimento do retângulo é 12 unidades e a largura é 5 unidades. 


\subsection{A solução atual do Problema Babilônio}

O empenho dos alunos para resolver este problema, no ambiente de aprendizagem da modelagem, é uma situação útil para o entendimento da externalização do conhecimento matemático tácito e da internalização do conhecimento matemático explícito.

Então, para realizar essa tarefa, os alunos tentarão extrair do conhecimento matemático tácito, que acumularam durante as suas experiências escolares, as informações e os conceitos matemáticos que serão utilizados no processo de resolução desta situação-problema. Para isso, eles selecionarão os métodos necessários para que eles possam externalizar o conhecimento matemático tácito e, dessa forma, sejam bem sucedidos na realização dessa tarefa. Assim, os alunos poderão acessar o conhecimento matemático tácito que possuem raciocinando da seguinte maneira:

Como o comprimento $(C)$ do retângulo excede a sua largura em sete unidades, temos a equação I que é dada por $C=L+7$. Como a área (A) do retângulo é de 60 unidades quadradas, temos a equação II que é dada por $C \cdot L=60$. Então, substituindo a equação I na equação II, uma nova equação será produzida, ou seja, $(L+7) \bullet L=60 \Rightarrow L^{2}+7 L=60 \Rightarrow L^{2}+7 L-60=0$. Para resolver esta equação, é necessária a utilização da fórmula de Bhaskara, ou seja, $\quad L=\frac{-7 \pm \sqrt{49+240}}{3} \Rightarrow L=\frac{-7+\sqrt{289}}{2} \Rightarrow L=\frac{-7+17}{2} \Rightarrow L=5$.

Substituindo $L=5$ na equação I, temos que. Portanto, podemos perceber que as mesmas respostas foram obtidas nos dois métodos que foram utilizados na resolução do problema proposto.

\subsection{Traduzindo e modelando}

Por outro lado, os alunos com o auxílio do professor como mediador do processo de ensino-aprendizagem, no ambiente de aprendizagem da modelagem, poderão traduzir e modelar o método atual e o método babilônio que foram utilizados para a resolução do problema proposto.

Por exemplo, no problema babilônio, as seguintes informações estão disponíveis para os alunos:

1) A diferença entre as medidas das duas dimensões.

2) A área da figura geométrica. 
Nesse caso, de acordo com Rosa (2007), os alunos poderão modelar ambos os métodos, utilizando as variáveis $d$ para representar a diferença entre o comprimento e a largura da figura geométrica e $A$ para representar a área do retângulo. No entanto, para realizar esta tarefa, alunos deverão disponibilizar o conhecimento tácito que possuem para acessar as informações necessárias para a elaboração de um modelo matemático que traduza a situação-problema proposta.

\subsection{Modelando o método atual}

Se C e L são o comprimento e a largura do terreno, temos:

I) $L=C+d$

II) $L \bullet C=A$

Substituindo a equação I na equação II, temos:

$(C+d) \bullet C=A \Rightarrow C^{2}+C d-A=0$

Utilizando a fórmula de Bhaskara, temos que:

$C=\frac{-d \pm \sqrt{d^{2}-4 \cdot 1 \bullet A}}{2 \cdot 1} \Rightarrow C=\frac{-d \pm \sqrt{d^{2}-4 A}}{2} \Rightarrow C=\frac{-d+\sqrt{d^{2}+4 A}}{2}$

Substituindo C em I, determinamos a largura.

$L=C+d \Rightarrow L=\frac{-d+\sqrt{d^{2}+4 A}}{2}+d \Rightarrow L=\frac{-d+\sqrt{d^{2}+4 A}+2 d}{2} \Rightarrow L=\frac{d+\sqrt{d^{2}+4 A}}{2}$

\subsection{Modelando o método babilônio}

A modelação do método babilônio constitui uma atividade interessante, motivadora e desafiadora para que os alunos verifiquem porque esta prática matemática funciona na resolução de problemas relacionados com áreas de quadrados e retângulos (ROSA, 2007). Porém, para que isso ocorra, os alunos devem acessar o conhecimento matemático tácito que possuem para que eles possam entender as seguintes etapas:

a) Computar a metade da diferença entre as duas dimensões, ou seja, $\frac{d}{2}$.

b) Elevar o resultado, obtido na etapa $a$, ao quadrado, ou seja, $\left(\frac{d}{2}\right)^{2}=\frac{d^{2}}{4}$.

c) Adicionar a área da figura ao resultado obtido na etapa $b$, ou seja, 


$$
\frac{d^{2}}{4}+A \Rightarrow \frac{4 A+d^{2}}{4} \text {. }
$$

d) Determinar a raiz quadrada do resultado obtido na etapa $c$, ou seja,

$$
\sqrt{\frac{4 A+d^{2}}{4}} \Rightarrow \frac{\sqrt{4 A+d^{2}}}{2} \text {. }
$$

e) Determinar a largura, adicionando a metade da diferença ao resultado obtido na etapa $d$ ou seja, $L=\frac{d}{2}+\frac{\sqrt{4 A+d^{2}}}{2} \Rightarrow L=\frac{d+\sqrt{4 A+d^{2}}}{2}$.

f) Determinar o comprimento, subtraindo a metade da diferença do resultado obtido na etapa $d$, ou seja, $C=-\frac{d}{2}+\frac{\sqrt{4 A+d^{2}}}{2} \Rightarrow C=\frac{-d+\sqrt{4 A+d^{2}}}{2}$.

A resolução desta situação-problema no ambiente de aprendizagem da modelagem permite que os alunos percebam que o método retórico utilizado pelos babilônios é uma derivação da fórmula de Bhaskara, que também pode ser obtida através do método de completar quadrados (ROSA, 2007). Nesta atividade, os alunos podem ser considerados como personagens ativos na construção do conhecimento a partir de relações interpessoais (interação social com o professor e com os demais alunos) e intrapessoais (interação dos alunos com eles próprios) que ocorrem no ambiente de aprendizagem da modelagem. Então, através da elaboração de modelos, os alunos podem internalizar o conhecimento explícito que eles adquiriram sobre o relacionamento da resolução de equações de segundo grau pela fórmula de Bhaskara com o método de completar quadrados.

Neste contexto, Kitcher (1984), Ernest (1998b) e Wenger (1998) especificam que para resolver uma determinada situação-problema, é importante reconhecermos a existência dos conhecimentos explícito e tácito na construção do conhecimento matemático, pois o processo de conversão entre estes conhecimentos emerge no ambiente de aprendizagem da modelagem.

\section{Considerações finais}

O conhecimento matemático é parte integrante da sociedade porque é influenciado pela atividade humana (D’AMBROSIO, 1990; ERNEST, 1998a; FITZSIMONS, 2002). De acordo com esta perspectiva, o conhecimento 
matemático é um produto de natureza social, pois envolve as ideias e as práticas matemáticas formais e informais que estão presentes nas atividades cotidianas dos diferentes grupos culturais que formam a sociedade contemporânea. Então, a natureza social da matemática é estabelecida pelos significados que são derivados do contexto sociocultural na qual ela se origina, pois esta característica da matemática está diretamente relacionada com os conhecimentos matemáticos tácito e explícito e com as práticas matemáticas desenvolvidas em cada grupo cultural. Consequentemente, a construção do conhecimento matemático está fundamentada nas ideias, nos conceitos, nos procedimentos e nas práticas matemáticas que são tácita e explicitamente utilizadas e negociadas pelos elementos destes grupos sociais para elaborar significados matemáticos específicos, com o objetivo de entender e compreender os fenômenos que são vivenciados diariamente.

De acordo com este contexto, o ambiente de aprendizagem da modelagem possui um papel importante para a construção do conhecimento matemático, pois pode facilitar a comunicação entre os professores e os alunos num ambiente propício para a conversão entre os conhecimentos matemáticos tácito e explícito. Então, a modelagem pode favorecer um ambiente de aprendizagem no qual os professores e os alunos constroem, compartilham e convertem os conhecimentos explícito e tácito através das interações sociais. Nesse processo de construção do conhecimento matemático, os conhecimentos matemáticos tácito e explícito dos professores e dos alunos se transformam, pois eles constroem significados matemáticos ao converter esses conhecimentos com a elaboração de modelos que possibilitam a produção de novas formas de conhecimento matemático. No ambiente de aprendizagem da modelagem, os conhecimentos explícito e tácito são compartilhados e convertidos através das interações sociais e com a utilização da experiência vivenciada e do conhecimento prévio que os professores e alunos trazem para esse ambiente. Assim, esse ambiente se caracteriza pelo entendimento e compreensão dos sistemas retirados da realidade, pois a reflexão e a análise crítica sobre a realidade auxiliam os alunos a converterem os conhecimentos explicito e tácito mediante o estudo formalizado dos modelos matemáticos que representam estes sistemas.

Nesta perspectiva, o ambiente de aprendizagem da modelagem pode ser considerado como um espaço educacional que favorece a deflagração da intervenção pedagógica do processo de ensino-aprendizagem em matemática, pois, nesse ambiente, os professores, como mediadores do processo educacional, auxiliam os alunos a externalizarem o conhecimento matemático tácito através de atividades pedagógicas contextualizadas, como por exemplo, a elaboração 
de modelos matemáticos. Isso significa que as atividades matemáticas desenvolvidas e os conceitos aprendidos através do ambiente de aprendizagem da modelagem introduzem novos modos de operação intelectual; por exemplo, abstrações e generalizações mais amplas acerca da realidade, que visam transformar os modos de utilização dos conhecimentos matemáticos tácito e explícito no ambiente acadêmico. Como consequência, de acordo com Rego (1996), na medida em que os alunos expandem a dimensão dos conhecimentos matemático tácito e explícito que possuem, eles começam a modificar o relacionamento cognitivo que dominam com o mundo que os cerca.

Entendemos que este processo deflagra o Ciclo Dambrosiano de construção do conhecimento, no qual a partir da realidade e por meio de ações transformadoras, permite que os alunos processem as informações e definam as estratégias necessárias para a solução de situações-problema enfrentadas no cotidiano. A Figura 6 mostra o Ciclo Dambrosiano da aquisição do conhecimento.

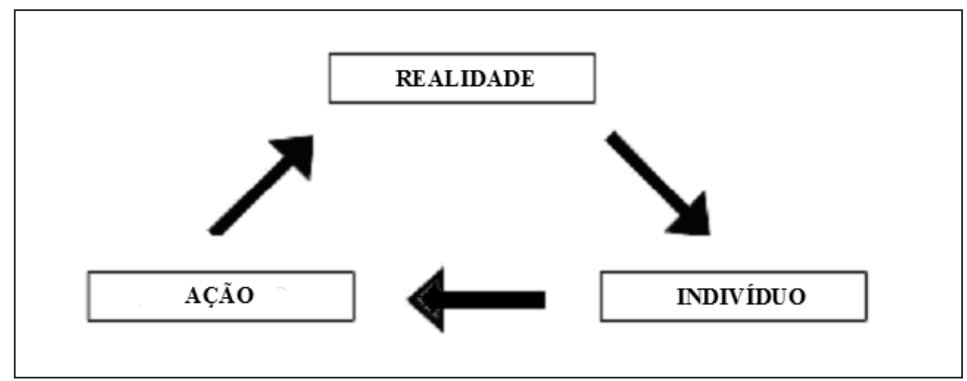

Figura 6 - Ciclo Dambrosiano de aquisição do conhecimento

Neste ciclo, as ações transformadoras resultam das estratégias motivadas pela necessidade que os alunos têm para conviver com a realidade, transformando-a através da análise, da reflexão e da previsão que eles realizam sobre os sistemas estudados com a utilização do ambiente de aprendizagem da modelagem como mediadora do processo de ensino-aprendizagem em matemática.

\section{Referências}

ALMEIDA, L. M. W.; DIAS, M. R. Um estudo sobre o uso da modelagem matemática como estratégia de ensino aprendizagem. Bolema, Rio Claro, SP, v. 17, n. 22, p. 19 - 35, out., 2004. 
AUSUBEL, D. P.; NOVAK, J.; E HANESIAN, H. Educational psychology: a cognitive view. New York, NY: Holt, Rinehart and Winston, 1978.

AUSUBEL, D. P.; NOVAK, J.; E HANESIAN, H. Psicologia educacional. Rio de Janeiro, RJ: Interamericana, 1980.

BARBOSA, J. C. Modelagem matemática: concepções e experiências de futuros professores. 2001, 253 f. Tese (Doutorado em Educação Matemática) - Instituto de Geociências e Ciências Exatas, Universidade Estadual Paulista, Rio Claro, SP, 2001.

BARBOSA, J. C. Modelagem matemática e os futuros professores. In: REUNIÃO ANUAL DAANPED, 25., 2002, Caxambu, MG. Anais... Caxambu, MG: ANPED, 2002. 1 CD-ROM.

BARBOSA, J. C. A prática dos alunos no ambiente de modelagem matemática: o esboço de um framework. In: BARBOSA, J. C.; CALDEIRA, A. D.; ARAÚJO, J. L. (Orgs.). Modelagem Matemática na Educação Matemática Brasileira: Pesquisas e Práticas Educacionais. Recife, PE: SBEM, 2007, p. 161-174.

BASSANEZI, R. C. Ensino-aprendizagem com modelagem matemática. São Paulo, SP: Editora Contexto, 2002.

BECKMAN, T. A methodology for knowledge management. In: IASTED INTERNATIONAL CONFERENCE ON ARTIFICIAL INTELLIGENCEAND SOFT COMPUTING, ASC, 97 $7^{\text {th }}, 1997$, Banff, Canadá. Proceedings... Banff, Canadá: ACT Press, 1997, p. 29 - 32.

BIEMBENGUT, M. S. Modelagem matemática \& implicações no ensinoaprendizagem de matemática. Blumenau, SC: Edifurb, 2004.

BLOODGOOD, J. M.; SALISBURY, W. D. Understanding the influence of organizational change strategies on information technology and knowledge management strategies. Decision Support Systems, Amsterdam, The Netherlands, v. 31, n. 1, p. 55 - 69, May. 2001.

BURAK, D. Modelagem matemática: Ações e interações no processo de ensinoaprendizagem.1992, 329 f. Tese (Doutorado em Psicologia Educacional) - Faculdade de Educação, Universidade Estadual de Campinas, Campinas, 1992.

CHAVES, S. N. A construção coletiva de uma prática de formação de professores de ciências: tensões entre o pensar e o agir. 2000. Tese (Doutorado em Educação) Faculdade de Educação, Universidade Estadual de Campinas, Campinas, 2000. 
CHOI, B.; LEE, H. Knowledge management strategy and its link to knowledge creation process. Expert System with Applications, London, England, v. 23, n. 3, p. 173 - 187, 2002.

CHOO, C. W. The knowing organization: how organizations use information to construct meaning, create knowledge and make decisions. International Journal of Information Management, Oxford, England, v. 16, n. 5, p. 329 - 340, 1996.

CHOO, C. W. The Knowing Organization. Oxford, England: Oxford University Press, 1998.

CONSWAY, B.; WHITTINGHAM, V. Managing knowledge and learning at unipart. Knowledge Management Review, London, England, v. 4, n. 3, p. 14 - 17, July/Aug. 2001.

D’AMBROSIO, U. Etnomatemática. São Paulo, SP: Editora Ática, 1990.

D'AMBROSIO, U. Etnomatemática: um programa. A Educação Matemática em Revista, Blumenau, SC, v. 1, n. 1, p. 5 - 11, 1993.

ERNEST, P. Social Constructivism as a Philosophy of Mathematics. Albany, NY: State University of New York Press, 1998a.

ERNEST, P. Mathematical knowledge and context. In: WATSON, A. (Ed.). Situated Cognition and the Learning of Mathematics. Oxford: University of Oxford Department of Educational Studies, 1998b, p. 13 - 29.

FITZSIMONS, G. E. What counts as mathematics? Technology of power in adult and vocational education. Dordrecht, Netherlands: Kluwer Academic Publisher, 2002.

FLEMMING, D. M.; LUZ, E.; COLLAÇO DE MELLO, A. C. Tendências em educação matemática. Palhoça, RS: UnisulVirtual, 2005.

FORD, K. M. et al. ICONKAT: an integrated constructivist knowledge acquisition tool. Knowledge Acquisition, v. 3, n. 2, p. 215 - 236, June 1991.

FRADE, C. The tacit-explicit dynamic in learning processes. In: CONFERENCE OF THE INTERNATIONAL GROUPFOR THE PSYCHOLOGY OF MATHEMATICS EDUCATION, $28^{\text {th }}, 2004$, Bergen, Norway. Proceedings... Bergen, Norway: PME, 2004. p. $407-414$. 
GILBERT, J. K.; BOULTER, C. J.; ELMER, R. Positioning models in science education and in design and Technology education. In J. K. GILBERT; C. J. BOULTER (Eds.), Developing models in science education. Dordrecht, The Netherlands: Kluwer Academic Publishers, p.3 - 17, 2000.

GRECA, I. M.; MOREIRA, M. A. Mental, physical, and mathematical models in the teaching and learning of physics. Science Education, Malden, MA, USA, v. 86, n. 1, p. $106-121,2002$.

JOSEPH, G. G. The crest of the peacock: Non-European roots of mathematics.

Princeton, NJ: Princeton University Press, 2000.

KERMALLY, S. Effective knowledge management: a best practice blueprint. New York, NY: John Wiley \& Sons, 2002.

KITCHER, P. The nature of mathematical knowledge. Oxford: Oxford University Press, 1984.

KNIJNIK, G. Exclusão e Resistência: educação matemática e legitimidade cultural. Porto Alegre: Editora Artes Médicas, 1996.

KOGUT, B.; ZANDER, U. Knowledge of the firm combinative capabilities and the replication of technology. Organization Science, Rochester, NY, USA, v. 3, n. 3, p. 383 $-397,1992$.

LEONARD-BARTON, D.; SENSIPER, S. The Role of tacit knowledge in group innovation. California Management Review, Berkeley, CA, v. 40, n. 3, p.112 - 127 , 1998.

LOPEZ, A. M.; DONLON, J. J. Knowledge engineering and education. Educational Technology, Englewood Cliffs, NJ, v. 41, n. 2, p. 45 - 50, Mar./Apr. 2001.

MALHOTRA, Y. Knowledge management and new organization forms: a framework for business model innovation. Information Resources Management Journal, Hershey, PA, v. 13, n. 1, p. 5 - 14, Jan./Mar. 2000.

MCLNERNEY, C. Knowledge management and the dynamic nature of knowledge. Journal of the American Society for Information Science and Technology, Hoboken, NJ, USA, v. 53, n. 12, p. 1009 - 1018, 2002.

MICHAELSON, R.; MICHIE, D.; BOULANGER, A. The technology of expert systems. Byte, Columbus, OH, USA,v. 10, n. 4, p. 303 - 312, Apr. 1985. 
MORETTO, V. P. Construtivismo: a produção do conhecimento em aula. Rio de Janeiro, RJ: DP \& A, 2003.

NELSON, R. R.; WINTER, S. G. An evolutionary theory of economic change. Cambridge, MA: Belknap Press of Harvard University Press, 1982.

NONAKA, I. The knowledge creating Company. Harvard Business Review, Cambridge, MA, USA, v. 69, n. 1, p. 96 - 104, Nov./Dec. 1991.

NONAKA, I. A dynamic theory of organizational knowledge creation. Organization Science, Hanover, MD, v. 5, n. 1, p. 14 - 37, Feb. 1994.

NONAKA, I.; TAKEUCHI, H. The knowledge-creating company. New York, NY: Oxford University Press, 1995.

NONAKA, I.; TAKEUCHI, H. A theory of organizational knowledge creation. International Journal of Technology Management, Oxford, England, v. 11, n. 7 - 8, p. 833 - 845, 1996.

NONAKA, I.; TAKEUCHI, H. Criação do conhecimento na empresa: como as empresas japonesas geram a dinâmica da inovação. Rio de Janeiro, RJ: Elsevier, 1997.

NONAKA, I.; TOYAMA, R.; KONNO, N. S. Ba and leadership: a unified model of dynamic knowledge creation. Long Range Planning, Stanford, CA, USA, v. 33, n. 1, p. $5-34,2000$.

ORNEK, F. Models in science education: Applications of models in learning and teaching science. International Journal of Environmental and Science Education, Bolu, Turkey, v. 3, n. 2, p. 35 - 45, Apr. 2008.

POLANYI, M. Knowing and being. Chicago, IL: Chicago University Press, 1969.

PORTIER, S. J.; WAGEMAS, L. J. J. M. The assessment of prior knowledge profiles: a support for independent learning? Distance Education, Stanhope Gardens NSW 2768, Australia, v. 16, n. 1, p. 65 - 87, 1995.

REGO, J. M. Retórica na Economia: Ideias no lugar. São Paulo, SP: Editora 34, 1996.

ROSA, M. From reality to mathematical modeling: A proposal for using ethnomathematical knowledge. 205f. Dissertação (Mestrado em Educação), College of Education, California State University, Sacramento, EUA, 2000.

ROSA, M. Currículo e matemática: algumas considerações na perspectiva etnomatemática. Plures Humanidades, Ribeirão Preto, SP, v. 6, n. 6, p. 81 - 96, 2005. 
ROSA, M. Um problema babilônio. Revista do Professor de Matemática, São Paulo, SP, v. 62, n. 1, p. 21 - 23, 2007.

ROSA, M.; OREY, D. C. Vinho e queijo: etnomatemática e modelagem! Bolema, Rio Claro, v. 16, n. 20, p. 1 - 16, set. 2003.

ROSA, M.; OREY, D. C. A dimensão crítica da modelagem matemática: Ensinando para a eficiência sociocrítica. Horizontes, Bragança Paulista, SP, v. 25, n. 2, p. 197 - 206, jul. - dez. 2007.

ROSA, M.; OREY, D. C. Ethnomathematics and cultural representations: Teaching in highly diverse contexts. Acta Scientiae, Canoas, RS, v. 10, n. 2, p. 27 - 46, jul. - dez. 2008.

ROSKA, T. Information based society and knowledge. In: WOLD SCIENCE FORUM, 1st, 2003, Budapest, Hungary. Proceedings... Budapeste, Hungria: WSF, 2003.

SKOVSMOSE, O. Cenários para investigação. Bolema, Rio Claro, v. 13, n. 14, p. 66 91,2000 .

SORENSEN, C.; LUNDH-SNIS, U. Innovation through knowledge codification. Journal of Information Technology, Hampshire, England, v. 16, n. 2 , p. 83 - 97, 2001.

TRENTIN, G. From formal training to communities of practice via network-based learning. Educational Technology, Englewood Cliffs, NJ, USA, v. 41, n. 2, p. 5 - 14, 2001.

TURBAN, E. Why expert systems succeed and why they fail. In: TURBAN, E.;

LIEBOWITZ, J. (Eds.). Managing Expert Systems. Hershey, PA: IGI Publishing, 1992, p. 2 - 13.

WENGER, E. Communities of Practice: Learning, Meaning and Identity. Cambridge: England, Cambridge University Press, 1998.

Submetido em Julho de 2010. Aprovado em Novembro de 2010. 